\title{
Secondary Hyperparathyroidism Presenting with Vocal Cord Paralysis
}

\author{
${ }^{1}$ Thomas WT Ho, ${ }^{2}$ Todd P McMullen \\ ${ }^{1}$ Endocrine Surgery, Department of General Surgery, Tan Tock Seng Hospital, Singapore \\ ${ }^{2}$ Endocrine Surgery, Department of Surgery, University of Alberta, Canada
}

Correspondence: Thomas WT Ho, Endocrine Surgery, Department of General Surgery, Tan Tock Seng Hospital, 33 Jalan Tan Tock Seng, 308433, Singapore, Phone: 65-6357-7807, Fax: 65-6357-7809, e-mail: thomasho95@gmail.com

\section{ABSTRACT}

The association of vocal cord paralysis with tumors in the neck generally raises the suspicion of an underlying malignancy. We report the clinical course, imaging and operative findings in a patient with secondary hyperparathyroidism presenting with vocal cord paralysis. The related literature is also reviewed. A 45-year-old man with end-stage renal failure was awaiting surgical treatment for secondary hyperparathyroidism. Ultrasound of the neck demonstrated hypoechoic nodules consistent with enlarged parathyroid glands. Several months later, just before the scheduled date for parathyroid surgery, he developed increasing hoarseness and vocal fatigue. Direct laryngoscopy revealed an immobile left vocal cord (VC). Neck examination and further imaging did not reveal the source of VC palsy. A presumptive diagnosis of thyroid or parathyroid malignancy involving the recurrent laryngeal nerve (RLN) was made. Intraoperatively, four enlarged parathyroid glands were encountered. The left superior gland was seen stretching and displacing the RLN without evidence of direct invasion. This was successfully dissected off while preserving the RLN. The remaining three parathyroid glands were subsequently removed with autotransplantation of a parathyroid remnant. In addition, a left thyroid lobectomy and ipsilateral level VI dissection was performed. Final pathology revealed parathyroid hyperplasia and thyroid follicular adenoma. No malignancy was seen. Three weeks later, the patient's biochemistry had normalized and his voice improved. Laryngoscopy confirmed a mobile left VC. Benign parathyroid tumors are rarely associated with RLN compression leading to VC paralysis. Such diagnoses are difficult to make preoperatively. In the absence of malignant invasion, the RLN should be preserved during surgery as it is likely to recover once the compression is relieved.

Keywords: Parathyroid, Benign, Hyperplasia, Hyperparathyroidism, Vocal cord paralysis.

\section{INTRODUCTION}

Secondary hyperparathyroidism (SHPT) is one of the major complications experienced by patients with chronic renal failure (CRF). The pathophysiology of SHPT is hallmarked by hyperplasia of all four parathyroid glands. Many patients with SHPT are asymptomatic and will only have abnormalities detectable by laboratory and radiographic studies. Laboratory investigations typically reveal an elevated intact parathyroid hormone (PTH) and phosphate level with a raised calciumphosphate product, raised alkaline phosphatase (ALP), and hypo- or normocalcemia. Untreated SHPT leads to progressive bone disease, soft tissue and vascular calcifications. Vocal cord paralysis is not generally associated with SHPT.

In this report, we discuss a patient with SHPT who presented with unilateral vocal cord paralysis due to recurrent laryngeal nerve (RLN) compression from a hyperplastic parathyroid gland. We also perform a literature review of benign parathyroid tumors associated with vocal cord paralysis.

\section{CASE REPORT}

A 45-year-old man with end-stage renal failure secondary to IgA nephropathy was assessed for suitability of surgery for SHPT in December 2009, due to inability to control his calcium, phosphorus and PTH levels. He had been on peritoneal dialysis and was waitlisted for a kidney transplant. His most recent laboratory studies in August 2009 revealed a serum PTH of $226 \mathrm{pmol} / \mathrm{l}$ (1.1-6.8), calcium of $2.49 \mathrm{mmol} / \mathrm{l}(2.1-2.6)$, phosphorus of $2.29 \mathrm{mmol} / \mathrm{l}(0.8-1.45)$, a calcium-phosphate product of 5.7, ALP of $107 \mathrm{u} / \mathrm{l}(30-130)$ and TSH of $0.67 \mathrm{mu} / \mathrm{l}(0.2-4.0)$. Relevant medications included optimal dosing of calcium carbonate and calcitriol. He was otherwise clinically asymptomatic. Other medical history included hypertension and obstructive sleep apnea. He was an ex-smoker and denied previous radiation exposure as well as a family history of malignancy. A sestamibi/SPECT scan performed before his surgical consultation in September 2009 showed increased focal uptake in the midpole of the left thyroid lobe suggestive of an intrathyroidal parathyroid gland. A neck ultrasound in October 2009 demonstrated two hypoechoic posterior nodules consistent with enlarged left $(2.4 \mathrm{~cm})$ and right $(1.5 \mathrm{~cm})$ superior parathyroid glands. The inferior parathyroids were however not seen. No thyroid masses were documented. The patient was consented and placed on a waitlist for subtotal parathyroidectomy. However, just before surgery in July 2010, the patient developed increasing hoarseness and vocal fatigue. Direct laryngoscopy revealed an immobile left vocal cord even though findings on neck examination remained unchanged from the previous consultation. A PET-CT scan failed to demonstrate either a central cerebral or peripheral cause 
of nerve palsy within the neck (Fig. 1) or thorax. Due to concerns for missing a thyroid malignancy, an US-guided biopsy was performed on the left-sided thyroid nodule. This yielded a highly cellular aspirate favoring a follicular neoplasm. At this juncture, a possible diagnosis of thyroid carcinoma involving the RLN was discussed with the patient and an additional consent for a possible left thyroid lobectomy with level VI neck dissection was obtained.

The patient underwent a neck exploration in October 2010. During the operation, four enlarged parathyroid glands were encountered. Both inferior glands were located low within the thyrothymic folds. The left superior gland measuring $2.5 \mathrm{~cm}$ was located just above the inferior thyroid artery and was intimately associated with the RLN posterior to it. This resulted in the nerve being stretched and displaced in a superolateral direction by the tumor although no invasion into the nerve was noted. Nevertheless, the parathyroid tumor appeared to be stuck onto the thyroid. In view of a possible parathyroid malignancy, a left thyroid lobectomy with ipsilateral central compartment nodal dissection was also performed. Removal of the remaining three enlarged parathyroid glands as well as a complete thymectomy was performed in view of SHPT. The left RLN was carefully dissected out and left intact. Finally, a remnant of parathyroid gland from the right side was reimplanted into the right sternocleidomastoid muscle. Subsequent histology of the specimens revealed nodular chief cell hyperplasia of all four parathyroid glands with no features of malignancy, hemorrhage or inflammation. Benign adenomatous nodules were seen in the thyroid lobe and reactive hyperplasia was noted in the lymph nodes. The patient's postoperative course was unremarkable and the anticipated bone hunger with his newly normal PTH level was managed with calcitriol and supplemental oral calcium. Upon review 3 weeks later in the clinic, the serum calcium and PTH levels had normalized. His voice had significantly improved and flexible laryngoscopy revealed left vocal cord movement indicating improving function.

\section{DISCUSSION AND REVIEW OF THE LITERATURE}

The majority, if not all patients with renal insufficiency will develop SHPT. Diffuse hyperplasia of the parathyroid gland is

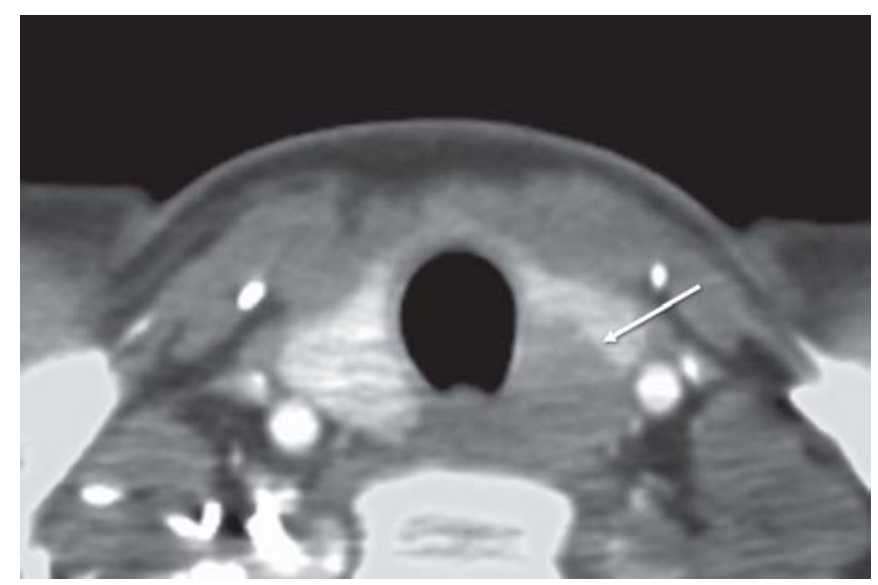

Fig. 1: Computed tomography of neck showing tumor posterior to left thyroid lobe initiated by hypocalcemia and phosphorus retention and becomes more severe as a result of 1, 25-dihydroxy vitamin D deficiency. As renal disease progresses, hyperplasia becomes nodular with asymmetrical enlargement of the gland. In later stages, parathyroid hyperplasia may escape the normal growth regulation with the emergence of an adenoma in one or more glands. ${ }^{1}$ Eventually, malignant transformation could occur, with sporadic reports in the literature of parathyroid carcinoma arising in the context of CRF. ${ }^{2}$

In general, patients who present with vocal cord paralysis and a mass in the trachea-oesophageal groove are considered to have a malignant neoplasm until proven otherwise. The differential diagnosis includes thyroid or parathyroid carcinoma, squamous cell carcinoma of the larynx or esophagus and metastatic disease from a distant primary cancer. ${ }^{3}$ In the case of parathyroid carcinoma, hoarseness occurs in up to $30 \%$ of patients due to tumor infiltration into the RLN. ${ }^{4}$ Benign parathyroid lesions presenting with vocal cord paralysis are rare. A survey of the English-language literature revealed only 13 cases including our own (Table 1)..$^{3,5-15}$ The cumulative underlying pathology in this review includes parathyroid cyst, adenoma and hyperplasia.

It is postulated that nerve paralysis resulting from benign parathyroid lesions arises from a combination of stretching, pressure or inflammation. Similar to malignant infiltration, the hoarseness is typically gradual in onset. However, a rapid onset of hoarseness is attributed to the presence of additional pathological process within the lesion resulting in an acute enlargement. Neck pain is often an associated symptom and provides an additional diagnostic clue. In our review, three patients had histological evidence of bleeding within the parathyroid tumor and one patient had evidence of an abscess. ${ }^{7,10,11,14,16}$ Some authors have speculated that the effect of hemodialysis on a well-perfused organ such as the parathyroid gland, or that hyperplasia itself may lead to internal bleeding in the absence of a coagulation disorder or trauma. ${ }^{7,14}$ Separately, the intermittent hoarseness associated with a parathyroid cyst is thought by some to be caused by fluctuations in the volume of cyst fluid. 9,12 $^{2}$

Apart from hoarseness, other clinical presentations in our review include hyperparathyroidism in 54\% (7 out of 13 patients), pain in 38\% (5 patients) and a neck mass in 15\% (2 patients). Angelos et al reported that hemorrhage into the parathyroid lesion may be associated with a hypercalcemic crisis or even a return to normocalcemia. ${ }^{10}$ It is noteworthy that the majority of patients in this review presented with a palpable neck mass on examination. Diagnostically, it can be challenging to be certain preoperatively whether the offending lesion is thyroid or parathyroid in origin, much less to differentiate a benign parathyroid lesion from a malignancy. US and CT have not enabled posterior thyroid nodules to be clearly distinguished from parathyroid nodules. ${ }^{15}$ Radionuclide scintigraphy provides no further help in the often-reported presence of cold nodules. ${ }^{13,15}$ Other than imaging, the use of fine needle aspiration (FNAC) with measurement of PTH level has been suggested as an additional diagnostic tool. ${ }^{9,12}$ Once again, 
Table 1: Reported cases of RLN palsy associated with benign parathyroid lesions

\begin{tabular}{|c|c|c|c|c|c|c|}
\hline Reference & Age/gender & Presentation & $\begin{array}{c}\text { Tumor } \\
\text { size }(\mathrm{cm})^{*}\end{array}$ & Pathology & $\begin{array}{l}R L N \\
\text { treatment }\end{array}$ & $\begin{array}{c}R L N \\
\text { recovery }\end{array}$ \\
\hline Current study & $45 \mathrm{M}$ & $2^{\circ} \mathrm{HPT}$, hoarseness & 2.5 & Hyperplasia & $\mathrm{P}$ & $\mathrm{Y}$ \\
\hline Lore et al, $1972^{5}$ & $55 \mathrm{M}$ & Hoarseness, pain & 2.0 & Adenoma & $\mathrm{S}$ & $\mathrm{N}$ \\
\hline Takimoto et al, $1989^{6}$ & $79 \mathrm{~F}$ & $1^{\circ} \mathrm{HPT}$, hoarseness & 2.5 & Adenoma & $P$ & $\mathrm{Y}$ \\
\hline Ben-Shlomo et al, $1990^{7}$ & $59 \mathrm{M}$ & Hoarseness, pain & 3.0 & Hyperplasia and hemorrhage & $\mathrm{P}$ & $\mathrm{Y}$ \\
\hline Coates et al, $1991^{8}$ & $60 \mathrm{M}$ & Hoarseness, mass & 8.0 & Cyst & $\mathrm{P}$ & $\mathrm{N}$ \\
\hline Grey et al, $1993^{9}$ & $36 \mathrm{~F}$ & Hoarseness, pain & 2.0 & Cyst & $P$ & $Y$ \\
\hline Angelos et al, $1997^{10}$ & $68 \mathrm{M}$ & $1^{\circ} \mathrm{HPT}$, pain, hoarseness & 1.5 & Adenoma and abscess & $\mathrm{P}$ & $\mathrm{Y}$ \\
\hline Iwasaki et al, $1999^{11}$ & $64 \mathrm{M}$ & $1^{\circ} \mathrm{HPT}$, hoarseness & 3.2 & Adenoma with hemorrhage & $\mathrm{P}$ & $\mathrm{Y}$ \\
\hline Sen et al, $2000^{12}$ & $37 \mathrm{~F}$ & Hoarseness & 2.5 & Cyst & $\mathrm{P}$ & Y \\
\hline Coelho et al, $2006^{13}$ & $49 \mathrm{M}$ & Hoarseness & 4.8 & Cyst & $P$ & $\mathrm{Y}$ \\
\hline Terada et al, $2007^{14}$ & $51 \mathrm{~F}$ & $2^{\circ} \mathrm{HPT}$, pain, hoarseness & 2.1 & Hyperplasia and hemorrhage & $\mathrm{P}$ & $\mathrm{N}$ \\
\hline Woo et al, $2008^{15}$ & $83 \mathrm{M}$ & Hoarseness, mass & 3.9 & Cyst & S & $\mathrm{N}$ \\
\hline Lee et al, $2009^{3}$ & $71 \mathrm{M}$ & $1^{\circ} \mathrm{HPT}$, hoarseness, dysphagia & 2.7 & Adenoma & $\mathrm{P}$ & Y \\
\hline
\end{tabular}

M: Male; F: Female; P: Preserved; S: Sacrificed; *Maximum dimension

FNAC may not differentiate between the two organs of origin, as was the case presented here, and cannot diagnose parathyroid carcinoma. In the review, only one patient had a presumed diagnosis of a parathyroid cyst. ${ }^{15}$ For the remaining patients, the preoperative diagnosis remained elusive with a high suspicion of carcinoma despite extensive preoperative workup.

Benign parathyroid tumors are characterized by the ability to excise the lesion in toto while preserving the integrity of the RLN. Only one author reported the need to sacrifice the RLN which was entering a cyst wall. ${ }^{15}$ Nerve paralysis caused by neuropraxia will often regain normal peripheral nerve conduction once the stretching or pressure from the offending benign tumor is relieved as observed in our case. Nerve recovery following axonotmesis is less likely since the nerve has undergone distal Wallerian degeneration and may not regrow to its target site. ${ }^{3}$ This is postulated to be the reason for failure of vocal cord recovery in two cases. ${ }^{8,14}$ It has been theorized that residual innervation through an intact RLN is important to provide neural tone to the cord and prevent it from atrophy and being replaced by fibrous tissue. ${ }^{8,17}$ Therefore, every effort should be made to surgically remove the nerve insult at the earliest opportunity and to ensure that the RLN remains intact at the end of the operation.

\section{CONCLUSION}

Unilateral vocal cord paralysis is usually attributed to a malignant cause, particularly in the presence of a neck mass. We report a case of SHPT presenting with vocal cord paralysis. Although this scenario is exceedingly rare, a benign parathyroid pathology should not be dismissed. In this context, the RLN has a favorable chance of recovery when the compression is relieved and should therefore be preserved during surgery.

\section{REFERENCES}

1. Lewin E, Huan J, Olgaard K. Parathyroid growth and suppression in renal failure. Semin Dial 2006;19:238-45.

2. Khan MW, Worcester EM, Straus FH, et al. Parathyroid carcinoma in secondary and tertiary hyperparathyroidism (2nd ed). J Am Coll Surg 2004;199:312-19.
3. Lee JC, Barkdull GC, Weisman RA. Parathyroid adenoma as a cause of vocal fold paralysis. Arch Otolaryngol Head Neck Surg 2009;135:712-13.

4. Fernandez-Ranvier GG, Jensen K, Khanafshar E, et al. Nonfunctioning parathyroid carcinoma: Case report and review of literature. Endocr Pract 2007;13:750-57.

5. Lore JM, Jr, Chakar SA, Montes M. Parathyroid adenomas causing vocal cord paralysis. Trans Am Acad Ophthalmol Otolaryngol 1972;76:1397-1401.

6. Takimoto $\mathrm{T}$, Okabe $\mathrm{Y}$, Ito $\mathrm{M}$, et al. Intravagal parathyroid adenoma. J Laryngol Otol 1989;103:704-06.

7. Ben-Shlomo I, Zohar S, Turani H, et al. Sudden dysphonia due to parathyroid apoplexy: A rare case of recurrent laryngeal nerve palsy. Head Neck 1990;12:355-56.

8. Coates G, Pearman K, Holl-Allen RT. Recurrent nerve palsy due to parathyroid cyst. Int Surg 1991;76:192-93.

9. Grey AB, Shaw JH, Anderson NE, et al. Parathyroid cyst with recurrent vocal cord paresis. Aust N Z J Surg 1993;63: 561-62.

10. Angelos P, Thompson NW, Giordano TJ. Spontaneous vocal cord paresis and return to normocalcemia: An unusual presentation of parathyroid adenoma with concomitant abscess. Surgery 1997;121:704-07.

11. Iwasaki K, Usami A, Oida I, et al. Sudden recurrent laryngeal nerve paralysis due to apoplexy of parathyroid adenoma. Auris Nasus Larynx 1999;26:101-04.

12. Sen P, Flower N, Papesch M, et al. A benign parathyroid cyst presenting with hoarse voice. J Laryngol Otol 2000;114: 147-48.

13. Coelho DH, Boey HP. Benign parathyroid cyst causing vocal fold paralysis: A case report and review of the literature. Head Neck 2006;28:564-66.

14. Terada T, Kawata R, Higashino M, et al. Sudden dysphonia due to spontaneous bleeding in secondary parathyroid hyperplasia. Arch Otolaryngol Head Neck Surg 2007;133:608-09.

15. Woo EK, Simo R, Conn B, et al. Vocal cord paralysis secondary to a benign parathyroid cyst: A case report with clinical, imaging and pathological findings (2008:6b). Eur Radiol 2008;18: 2015-18.

16. Bacourt F, Brun JG, Lacombe P, et al. Parathyroid adenoma disclosed by a massive subcapsular hemorrhage. Presse Med 1984;13:669-70.

17. Chi SY, Lammers B, Boehner H, et al. Is it meaningful to preserve a palsied recurrent laryngeal nerve? Thyroid 2008;18: 363-66. 